\title{
Discovery of a spectroscopic binary comprising two hot helium-rich subdwarfs ${ }^{\star}$
}

\author{
A. Ahmad ${ }^{1}$, C. S. Jeffery ${ }^{1}$, and A. W. Fullerton ${ }^{2,3}$ \\ 1 Armagh Observatory, College Hill, Armagh BT61 9DG, N. Ireland, UK \\ 2 Dept. of Physics and Astronomy, University of Victoria, PO Box 3055, Victoria, BC V8W 3P6, Canada \\ 3 Dept. of Physics and Astronomy, The Johns Hopkins University, 3400 North Charles Street, Baltimore, MD 21218, USA
}

Received 19 December 2003 / Accepted 17 January 2004

\begin{abstract}
Helium-rich subdwarf B (He-sdB) stars form a very small fraction of the population of hot subdwarf stars in our Galaxy. They have been found both in the field of our Galaxy as well as in globular clusters. The evolution of these rare stars has recently been the subject of much debate involving both binary and single star evolution models. We report our serendipitous discovery that the prototype - PG 1544+488 - is a binary containing two low-mass helium-rich hot subdwarfs. This discovery challenges existing evolutionary models for He-sdB stars and suggests that they may be formed through close binary evolution and following the ejection of a common envelope.
\end{abstract}

Key words. stars: chemically peculiar - stars: early-type - stars: subdwarfs - stars: individual: PG 1544+488 stars: fundamental parameters - stars: binaries: spectroscopic

\section{Introduction}

Helium-rich subdwarf B (He-sdB) stars are rare, hot subluminous stars found in the field of our Galaxy (Green et al. 1986) and in globular clusters (Moehler et al. 1997, 2002). Originally classified as sdOD stars in the Palomar-Green (PG) catalogue of faint blue stars (Green et al. 1986), He-sdB stars are spectroscopically identified as subdwarfs showing pure HeI absorption with very weak or absent hydrogen Balmer absorption lines in their optical spectra.

Two evolutionary models have been proposed for the formation of He-sdB stars. The first, originally proposed by Iben \& Tutukov (1986) and later modelled by Saio \& Jeffery (2000), involves the merger of two white dwarfs (WD) to form a He-sdB star. Our analysis of the optical spectra (Ahmad \& Jeffery 2003 - Paper I) and flux distributions (Ahmad \& Jeffery 2004 - Paper II) of He-sdB stars provides support for the white dwarf merger model. The second, proposed by Brown et al. (2001), involves convective flash mixing of the atmosphere of a star on the white dwarf cooling track which has previously undergone a phase of high mass loss. Lanz et al. (2004) have recently reported that their analysis of FUSE spectra of three He-sdB stars provides support for the "flash-mixing" model. The WD merger model predicts a single helium-rich subdwarf

Send offprint requests to: A. Ahmad, e-mail: amir@star.arm.ac.uk

$\star$ Based on observations made with the William Herschel and Isaac Newton Telescopes operated on the island of La Palma by the Isaac Newton Group in the Spanish Observatorio del Roque de los Muchachos of the Instituto de Astrofísica de Canarias and with the NASA-CNES-CSA Far Ultraviolet Spectroscopic Explorer.
B star whereas the flash mixing scenario may occur in a close binary, mass transfer providing the required mass loss.

High-resolution $(\lambda / \Delta \lambda \sim 15000)$ optical blue and red spectra of the He-sdB prototype PG 1544+488 were obtained over two consecutive nights in 2003 April to measure detailed chemical abundances. While carrying out the analysis, we noticed that some of the helium line profiles had an unusual shape. On closer examination it became evident that this was due to line doubling. Further investigation of these data including a re-extraction of the FUSE data, is described here. It has demonstrated that the only likely explanation is that PG 1544+488 is a short-period spectroscopic binary.

\section{Observations and data reduction}

\subsection{Optical spectroscopy}

Spectroscopic observations of PG $1544+488$ were carried out with the William Herschel Telescope (WHT: $4.2 \mathrm{~m}$ ) in 2003 April using the double beam ISIS spectrograph and 5400 dichroic. Blue spectra were obtained with the H2400B and R1200B gratings while the red spectra were obtained with the R1200R grating. Details of the observations are listed in Table 1.

The stellar spectra were bias subtracted, flat-fielded, wavelength calibrated and sky subtracted using standard IRAF procedures. $\mathrm{CuNe}+\mathrm{CuAr}$ arc lamps were used for wavelength calibration. The spectra were then normalized using a fifth order cubic spline function. The dichroic, which acts as a beam 
Table 1. Spectroscopic observations of PG $1544+488$.

\begin{tabular}{cccc}
\hline \hline Date & Telescope & \multicolumn{2}{c}{ Instrument } \\
Grating & $\lambda[\AA]$ & Exp. [s] & $R(\lambda / \Delta \lambda)$ \\
\hline 2002 04 30 & INT & \multicolumn{2}{c}{ IDS } \\
R1200B & $3900-5000$ & 600 & 4500 \\
\hline 2002 05 01 & INT & \multicolumn{2}{c}{ IDS } \\
R1200B & $3900-5000$ & 600 & 4500 \\
R1200B & $3900-5000$ & 600 & 4500 \\
\hline 2003 04 10 & WHT & \multicolumn{2}{c}{ ISIS } \\
H2400B & $3900-4280$ & 1800 & 18500 \\
R1200R & $6100-6730$ & 1800 & 14000 \\
H2400B & $4050-4450$ & 1800 & 19000 \\
R1200R & $6100-6730$ & 1800 & 14000 \\
H2400B & $4610-5000$ & 1800 & 21500 \\
R1200R & $6100-6730$ & 1800 & 14000 \\
\hline 2003 04 11 & WHT & \multicolumn{2}{c}{ ISIS } \\
R1200B & $4210-5000$ & 900 & 10000 \\
R1200R & $6100-6730$ & 900 & 14000 \\
R1200B & $4210-5000$ & 900 & 10000 \\
R1200R & $6100-6730$ & 900 & 14000 \\
H2400B & $4345-4730$ & 1400 & 20500 \\
R1200R & $6100-6730$ & 1400 & 14000 \\
H2400B & $4345-4730$ & 1000 & 20500 \\
R1200R & $6100-6730$ & 1000 & 14000 \\
\hline
\end{tabular}

splitter, has a response function which is not totally corrected by flat-fielding hence the high order spline had to be used.

The spectral coverage with the H2400B grating is $\sim 375 \AA$. To observe the full spectral coverage from 3900-5000 $\AA$ with sufficient overlaps between segments, four short wavelength regions were observed separately. The red spectra were obtained simultaneously with the blue spectra.

Radial velocities were also measured from the reduced optical blue spectra obtained at the Isaac Newton Telescope (INT: $2.5 \mathrm{~m}$ ) in 2002 April/May and described in Paper I.

\subsection{Far ultraviolet observations}

Far-ultraviolet observations of PG $1544+488$ carried out with the FUSE satellite were retrieved from Multimission Archive at the Space Telescope (MAST). The observations were made in the time-tag (TTAG) mode through the large aperture and are listed in Table 2. The spectra were re-reduced with CalFUSE 2.4.1 by using a custom script which split each exposure into short exposures of $\sim 900 \mathrm{~s}$ to allow us to measure radial velocities. A short section of the FUSE spectra is shown in Fig. 1.

As indicated in Table 2, PG 1544+488 (=FUSE target C12901, PI: T. M. Brown) was observed twice. The first observation (C1290101) was divided into two exposures (i.e., C1290101001 and C1290101002), while the second observation was divided into 4 exposures. Unfortunately, the "Side 2"
Table 2. FUSE observations of C12901 in the wavelength range 905-1188 .

\begin{tabular}{|c|c|c|c|}
\hline $\begin{array}{l}\text { Rootname } \\
\text { sub-exposure }\end{array}$ & UT date [start] & HJD [start] & $\begin{array}{l}\text { Nominal } \\
\text { Exp. [s] }\end{array}$ \\
\hline C1290101001 ${ }^{1}$ & 20020326 & 52360.2739 & 4091.3 \\
\hline 1 & & 52360.2739 & 900 \\
\hline 2 & & 52360.2843 & 900 \\
\hline 3 & & 52360.2947 & 900 \\
\hline 4 & & 52360.3052 & 900 \\
\hline 5 & & 52360.3156 & 491 \\
\hline $\mathrm{C} 1290101002^{1}$ & 20020326 & 52360.3527 & 3178.4 \\
\hline 1 & & 52360.3527 & 900 \\
\hline 2 & & 52360.3631 & 900 \\
\hline 3 & & 52360.3735 & 900 \\
\hline 4 & & 52360.3839 & 478 \\
\hline $\mathrm{C} 1290102001^{2}$ & 20020714 & 52469.9869 & 2264.6 \\
\hline 1 & & 52469.9869 & 900 \\
\hline 2 & & 52469.9973 & 900 \\
\hline 3 & & 52470.0078 & 464 \\
\hline C1290102002 ${ }^{2}$ & 20020714 & 52470.0551 & 2374.7 \\
\hline 1 & & 52470.0551 & 900 \\
\hline 2 & & 52470.0656 & 900 \\
\hline 3 & & 52470.0760 & 574 \\
\hline C1290102003² & 20020714 & 52470.1256 & 2288.8 \\
\hline 1 & & 52470.1256 & 900 \\
\hline 2 & & 52470.1360 & 900 \\
\hline 3 & & 52470.1465 & 488 \\
\hline C12901020042 & 20020714 & 52470.2018 & 1612.9 \\
\hline 1 & & 52470.2018 & 900 \\
\hline 2 & & 52470.2122 & 712 \\
\hline
\end{tabular}

${ }^{1}$ No spectra from LiF2A, LiF2B, SiC2A, and SiC2B as detector 2 was down.

${ }^{2}$ The target drifted from the LWRS aperture during exposure 001 and never returned. No useful LiF2A or LiF2B spectra.

observations were compromised in both cases, either by detector problems (C1290101) or alignment problems (LiF2 channel of C1290102).

These 6 exposures were further divided into sub-exposures, which were nominally $900 \mathrm{~s}$ long. This was accomplished by specifying "good time intervals" explicitly in the input "screening" files used by CalFUSE. So, for sub-exposure 1 of a given exposure, CalFUSE was told that everything beyond the first 900 s of the total integration was "bad". CalFUSE then processed only the "good" photons and produced a spectrum from the first $900 \mathrm{~s}$ of the total integration. For sub-exposure 2, CalFUSE processed only photons that arrived between 900 and $1800 \mathrm{~s}$ after the start of the exposure, and so on. Although this procedure was easy to script, the resultant sub-exposures were not always $900 \mathrm{~s}$ long because CalFUSE performs additional screening, in particular to look for short-duration spikes in the count rate known as "event bursts". Event bursts are 


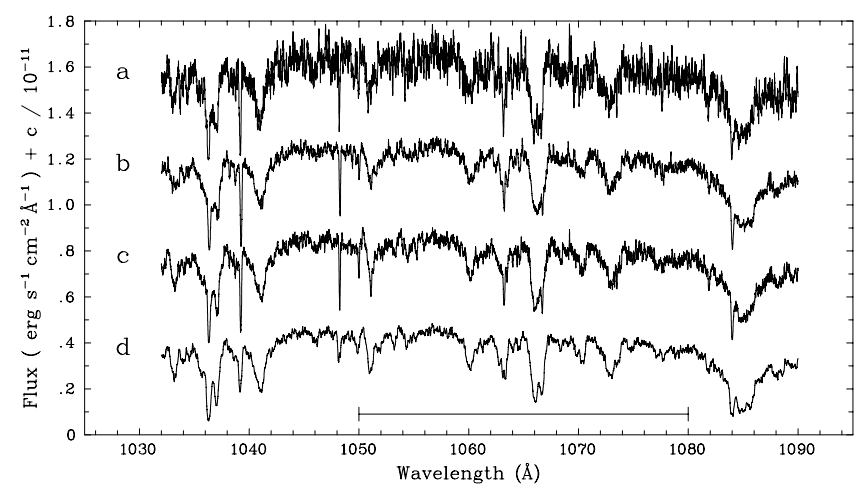

Fig. 1. Short segment of the FUSE spectra observed with SiC1A detector. a) $900 \mathrm{~s}$ exposure spectrum, b) Co-added spectra from $2002 \mathrm{March}$ FUSE observations, c) Co-added spectra from 2002 July FUSE observations, d) Sharpened template. The horizontal bar denotes the spectral region (1050-1080 ̊) used for cross-correlation.

believed to be extraneous, though their origin remains unknown. CalFUSE removes all photons that arrive during an event burst and decrements the exposure time accordingly. For some sub-exposures, this produced effective integration times that were less than the nominal value of $900 \mathrm{~s}$.

This divides the initial sample of 6 spectra into 20 spectra, which are distributed as shown in Table 2. Since each subexposure generates 8 spectra (LiF1A, SiC1A, LiF1B, SiC1B, LiF2A, SiC2A, LiF2B, SiC2B), this produced 160 FITS files. Unfortunately, there was no useful LiF2 data from either observation. During C1290101 the high-voltage to the detector was down; and during all but the very first part of C1290102 the LiF2 mirror was apparently mispositioned so that the target was not in the LWRS aperture. The unfortunate consequence is that this leaves little flux-calibrated information in the $1100-1187 \AA$ region, since the redundant $\mathrm{LiF} 1$ channel is compromised by the shadow of detector grid wires (an optical effect known as "the worm") that falls at $\sim 1150 \AA$. However it does leave us with 20 independent exposures from which radial velocity measurements can be made.

\section{Radial velocity measurements}

Radial velocities for PG 1544+488 were measured from spectra obtained with the FUSE satellite, the INT and the WHT. The errors quoted in Table 3 are estimated from the formal errors derived from the least-squares fit. The observations were made with different instruments/telescopes and velocities determined with different methods, so there are additional systematic errors in the radial velocity measurements and hence the quoted errors should be treated as the lower limit to the total error. The radial velocities from the WHT and FUSE spectra are plotted in Fig. 2.

\subsection{WHT spectra}

Radial velocities were determined from the WHT spectra by fitting multiple Gaussians to the cores of the strong helium lines listed in Table 4 using the method of least squares. We label the red component $\mathrm{A}$ and the blue component B (Fig. 3). For lines
Table 3. Radial velocities of PG $1544+488$ measured from farultraviolet and optical spectra.

\begin{tabular}{|c|c|c|}
\hline \multirow{2}{*}{$\begin{array}{c}\text { HJD } \\
-2400000 \\
\end{array}$} & \multicolumn{2}{|c|}{ Heliocentric $R V\left[\mathrm{~km} \mathrm{~s}^{-1}\right]$} \\
\hline & A & B \\
\hline \multicolumn{3}{|l|}{ FUSE } \\
\hline 52360.2791 & $-85.9 \pm 2.0$ & - \\
\hline 52360.2895 & $-73.2 \pm 22.6$ & - \\
\hline 52360.3000 & $-77.9 \pm 9.7$ & - \\
\hline 52360.3104 & $-75.3 \pm 4.4$ & - \\
\hline 52360.3185 & $-59.8 \pm 23.0$ & - \\
\hline 52360.3579 & $-39.8 \pm 25.7$ & - \\
\hline 52360.3683 & $-40.3 \pm 16.9$ & - \\
\hline 52360.3787 & $-40.9 \pm 13.6$ & - \\
\hline 52360.3867 & $-25.5 \pm 28.8$ & - \\
\hline \multicolumn{3}{|l|}{$\mathrm{INT}^{1}$} \\
\hline 52394.2513 & \multicolumn{2}{|c|}{$-18.4 \pm 10.1$} \\
\hline 52395.2466 & \multicolumn{2}{|c|}{$-6.9 \pm 14.2$} \\
\hline 52395.2503 & \multicolumn{2}{|c|}{$-19.4 \pm 17.4$} \\
\hline \multicolumn{3}{|l|}{ FUSE } \\
\hline 52469.9921 & $-13.9 \pm 22.4$ & - \\
\hline 52470.0026 & $-4.7 \pm 37.3$ & - \\
\hline 52470.0105 & $-12.5 \pm 17.2$ & - \\
\hline 52470.0604 & $-34.8 \pm 22.1$ & - \\
\hline 52470.0708 & $-33.5 \pm 29.5$ & - \\
\hline 52470.0793 & $-28.9 \pm 33.1$ & - \\
\hline 52470.1308 & $-75.8 \pm 22.2$ & - \\
\hline 52470.1413 & $-61.4 \pm 36.7$ & - \\
\hline 52470.1493 & $-77.8 \pm 23.4$ & - \\
\hline 52470.2070 & $-91.7 \pm 28.4$ & - \\
\hline 52470.2163 & $-97.0 \pm 37.5$ & - \\
\hline \multicolumn{3}{|l|}{ WHT } \\
\hline 52740.6955 & $38.7 \pm 2.2$ & $-117.4 \pm 2.5$ \\
\hline 52740.7232 & $25.5 \pm 3.5$ & $-102.6 \pm 2.8$ \\
\hline 52740.7503 & $-8.6 \pm 9.2$ & $-76.7 \pm 11.4$ \\
\hline 52741.6809 & $43.0 \pm 15.4$ & $-119.0 \pm 6.6$ \\
\hline 52741.6913 & $38.6 \pm 16.2$ & $-112.6 \pm 8.2$ \\
\hline 52741.7420 & $2.6 \pm 18.3$ & $-76.4 \pm 3.7$ \\
\hline 52741.7559 & $-2.3 \pm 20.2$ & $-69.6 \pm 2.7$ \\
\hline
\end{tabular}

${ }^{1}$ See Sect. 3.2.

which showed two components (i.e. HeI $4921 \AA$ and $6687 \AA$ ), a triple Gaussian was fitted in which the first Gaussian $(\mathrm{Gf}(1)$ : Fig. 4) fits the broad wings of the line, the second $(\operatorname{Gf}(2))$ and third $(\mathrm{Gf}(3))$ fit the two narrow components of the double profile. Their positions gave the radial velocities of both components, $v_{\mathrm{A}}$ and $v_{\mathrm{B}}$. The full width half maxima, $w_{\mathrm{A}}$ and $w_{\mathrm{B}}$, were also noted. When the two components are not fully resolved, $w_{\mathrm{A}}$ and $w_{\mathrm{B}}$ are used as fixed inputs to the triple Gaussian fit, solving only for their intensities and positions (or velocities). The principle is illustrated in Fig. 4. Radial velocities are 

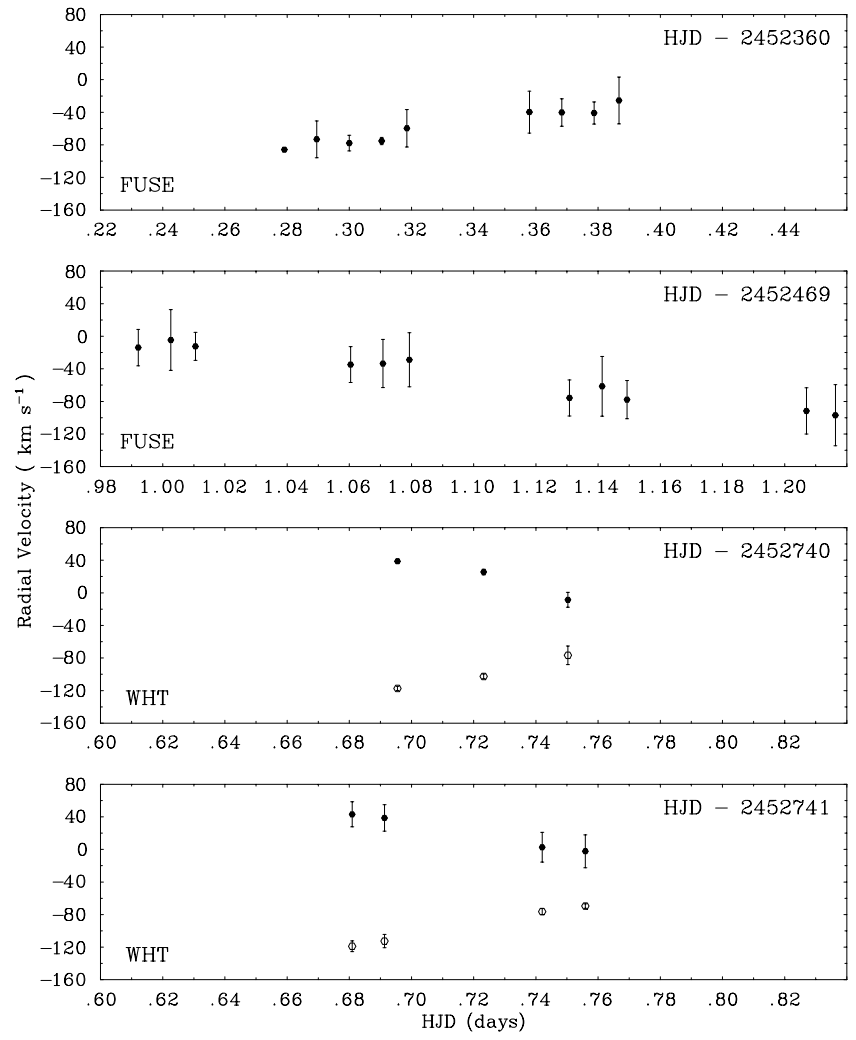

Fig. 2. Radial velocities derived from the FUSE and WHT observations. The filled symbols represent velocities for the stellar component $\mathrm{A}$ while the open symbols represent velocities for the stellar component B.

Table 4. Lines used in the optical blue and red spectra to measure radial velocities of the stellar components $\mathrm{A}$ and $\mathrm{B}$.

\begin{tabular}{ccccc}
\hline \hline HJD & HeI & HeII & HeI & HeI \\
& $4387.93 \AA$ & $4685.71 \AA$ & $4921.93 \AA$ & $6678.15 \AA$ \\
\hline 52740.6955 & - & - & - & $\mathrm{A} / \mathrm{B}$ \\
52740.7232 & $\mathrm{~A}$ & - & - & $\mathrm{A} / \mathrm{B}$ \\
52740.7503 & - & $\mathrm{A}$ & $\mathrm{A} / \mathrm{B}$ & $\mathrm{A} / \mathrm{B}$ \\
52741.6809 & $\mathrm{~A}$ & $\mathrm{~A}$ & $\mathrm{~A} / \mathrm{B}$ & $\mathrm{A} / \mathrm{B}$ \\
52741.6913 & $\mathrm{~A}$ & $\mathrm{~A}$ & $\mathrm{~A} / \mathrm{B}$ & $\mathrm{A} / \mathrm{B}$ \\
52741.7420 & $\mathrm{~A}$ & $\mathrm{~A}$ & - & $\mathrm{A} / \mathrm{B}$ \\
52741.7559 & $\mathrm{~A}$ & $\mathrm{~A}$ & - & $\mathrm{A} / \mathrm{B}$ \\
\hline
\end{tabular}

calculated from the measured line positions and the theoretical rest wavelengths.

In some lines there is no evidence of doubling at any time, even though it is seen in other lines at the same time. For such lines (i.e. HeI $4388 \AA$ and HeII $4686 \AA$ ) only a double Gaussian is fitted, one component to the broad wings and the other to the line core. Hence a radial velocity is derived only for component A.

Star A appears to be more luminous of the two and as one moves to shorter wavelengths, the observed stellar spectrum seems to be dominated by A (see Sect. 5). In cases where there are several measurements of velocities from different lines (Table 4), the mean velocity, weighted by the inverse of the

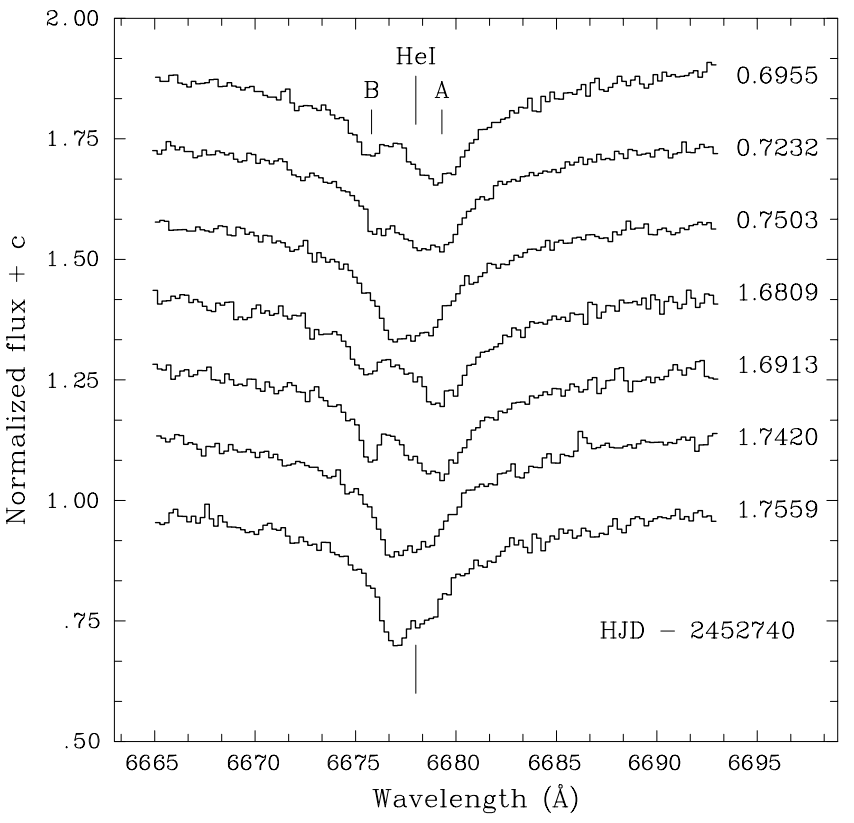

Fig. 3. Line profile variation in $\mathrm{PG} 1544+488$ seen here in the HeI $6678 \AA$ absorption line.

errors (weighted mean) was taken. The final velocities listed in Table 3 were obtained after correcting to the heliocentric frame.

\subsection{INT spectra}

Radial velocities were also calculated from three moderate resolution optical spectra (3900-5000 ̊) of PG 1544+488. These data were earlier used for measuring the effective temperature $\left(T_{\text {eff }}\right)$, surface gravity $(\log g)$ and helium-abundance $\left(n_{\mathrm{He}}\right)$ (Paper I). The normalized observed spectra were crosscorrelated with a theoretical blue spectrum using the Starlink package DIPSO (Howarth et al. 1998). The position of the peak of the resulting cross-correlation function (ccf) was measured again by fitting a Gaussian. This position was converted to a radial velocity shift and corrected to the heliocentric frame (Table 3). Owing to the lower resolution of the INT spectra, the components A and B are not resolved in either the ccf or in any individual helium line profiles. Since the ccf simply measures the shifts of the average line profile, it is blind to the presence of line doubling unless the components are well resolved. If two components are present, the measured velocities are a weighted average of their combined velocities, the weights depending on the relative contributions to the total flux at the wavelengths observed.

\subsection{FUSE spectra}

The measurement of radial velocities from the FUSE spectra was a challenge due to the presence of broad stellar absorption lines and several strong interstellar lines. The wavelength region 1050-1080 (Fig. 1) was selected for measuring the radial velocities because it is dominated by stellar lines. On FUSE, it is covered by four detectors, SiC1A, SiC2B, LiF1A and $\mathrm{LiF} 2 \mathrm{~B}$ providing independent measurements. 


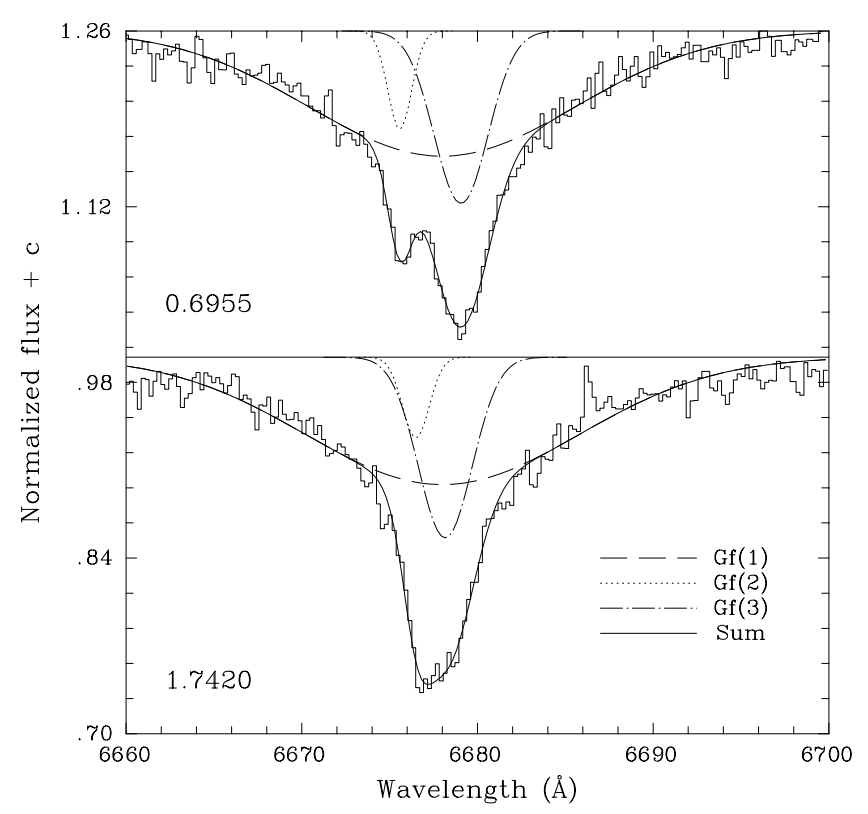

Fig. 4. The three components of the Gaussian fit to the HeI $6678 \AA$ absorption line profile. The top panel shows the Gaussian fit when the two components are resolved. The bottom panel shows the Gaussian fit when the two components are merged. The labels are the same as in Fig. 3.

Unfortunately we do not have any SiC2B and LiF2B spectra from the first FUSE observation. The LiF2B spectra from the second FUSE observation were also not suitable for the study (see Sect. 2.2). The first set of velocities were calculated from the $\mathrm{SiC} 1 \mathrm{~A}$ and LiF1A spectra. The second set of velocities were computed from the $\mathrm{SiC} 1 \mathrm{~A}, \mathrm{SiC} 2 \mathrm{~B}$ and $\mathrm{LiF} 1 \mathrm{~A}$ spectra.

The re-reduced spectra were co-added to make a template spectrum. This template was then normalized using a high order polynomial function. The same function was used to normalize the individual spectra. The ccf between the individual spectra and the template was computed and a velocity was measured by fitting a Gaussian function to the peak of the ccf. The individual short exposure spectra were then corrected with the estimated velocities and co-added once again to create the sharpened template shown in Fig. 1d. Radial velocities were again measured from the ccf between the spectra and the sharpened template. In cases where two or more independent measurements were available, a weighted mean was taken.

Since these radial velocities were relative to the sharpened template, correction to the heliocentric frame was obtained by cross-correlating the sharpened template with a theoretical spectrum and applying the appropriate heliocentric corrections (Table 3). A more detailed description of radial velocity measurement by cross-correlation and template sharpening can be found in Dudley \& Jeffery (1990). Note that the process of sharpening on the stellar velocities has the effect of smearing any (stationary) interstellar lines in the spectrum (Fig. 1d), thereby weakening their contribution in the ccf.
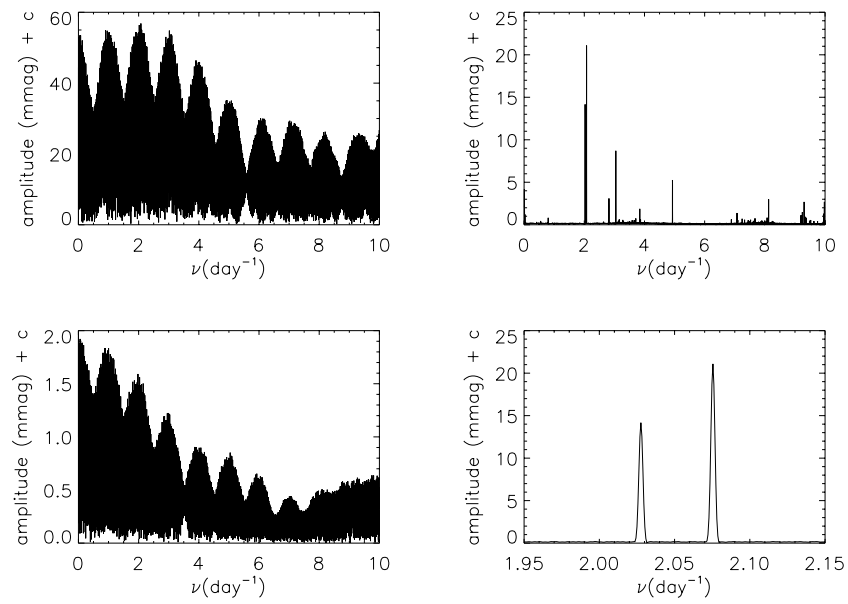

Fig. 5. The power spectrum of the spectroscopic binary PG 1544+488 from radial velocities obtained from FUSE and the WHT in 2002/2003. Top left: the discrete Fourier transform of the full dataset. Bottom left: the sampling function. Top right: the DFT after application of the CLEAN procedure. Bottom right: enlargement of the same plot.

\section{Orbital solution}

Several estimates of the period of PG $1544+488$ can be derived by a simple inspection of the data. For example the velocities of both components observed in the WHT data repeat almost exactly after $24 \mathrm{~h}$, so the period must be close to a unit fraction of one day. Secondly, one set of FUSE observations covers an interval of nearly $6 \mathrm{~h}$, and does not complete a full cycle, so the period must be greater than $1 / 4$ day. A period of one day does not provide a very good fit to the data, leaving only $1 / 2$ and $1 / 3$ day as reasonable alternatives. To distinguish between these alternatives, the Discrete Fourier Transform (DFT) of the radial velocities for the star visible in all FUSE and WHT observations was computed (Fig. 5). The velocities from the INT spectra were not used in the orbital solution since they have large systematic errors (see Sect. 3.2). The DFT of the sampling function (power window) is heavily contaminated by the cycles day ${ }^{-1}$ and the cycles year ${ }^{-1}$ aliases, but the highest peak in the power spectrum does occur near a frequency of 2 cycles day ${ }^{-1}$. The problem is almost ideal for the use of the CLEAN algorithm (Högbom 1974) because there should be only one true frequency present in the data. Other structure is due mostly to the sampling, with some leakage into side lobes due to random and systematic errors. Applying CLEAN to the data set using a gain of 0.5 and 100 iterations, we find a principal peak at 2.08 cycles day $^{-1}$, with weaker peak at 2.02 cycles day $^{-1}$.

A weakness of the CLEAN algorithm is that, in cases like these it may fail to identify the correct peak in the power spectrum, but may instead highlight an alias of the true period. Whatever method we use, the data currently available leave an uncertainty of the order of a few cycles year ${ }^{-1}$ in the derived orbital frequency.

In order to learn more, we have assumed a circular orbit and fitted the data for the star visible in all four data sets using a gradient expansion algorithm to compute a non-linear 


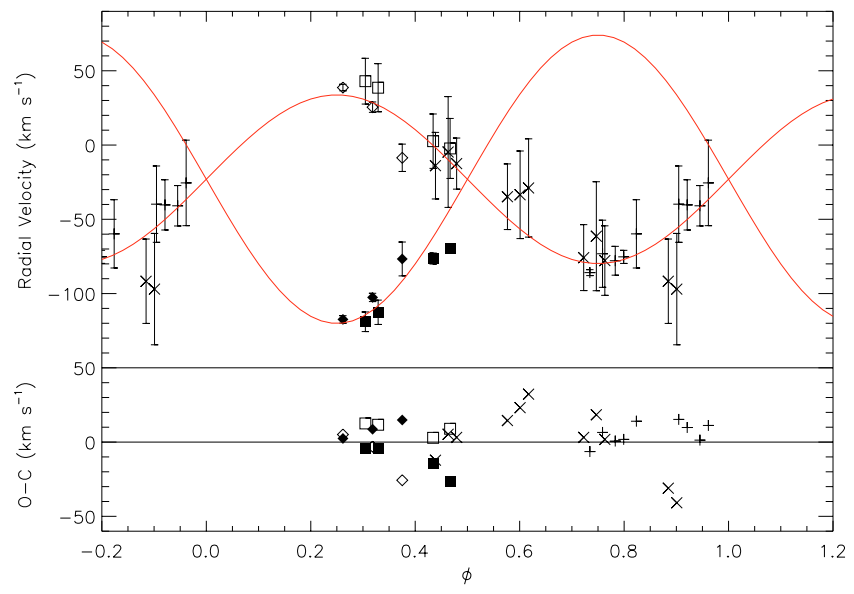

Fig. 6. Approximate orbital solution with orbital period $=0.48 \mathrm{~d}$ for PG $1544+488$. Observed data are shown for different dates: + FUSE (HJD 2452 360), $\times$ FUSE (HJD 2452 469), $\square$ WHT (HJD 2452740 ) and $\diamond$ WHT (HJD 2452741 ). Data for the secondary are shown as filled symbols. The best fit orbits are shown as smooth curve. Residuals to this fit are shown in the bottom panel.

weighted least squares fit, in which the systemic velocity $\gamma$, frequency $v_{\text {orb }}$, amplitude $k_{1}$ and phase $\phi$ are free parameters. A feature of using the least-squares approach is that it modifies any frequency selected from the DFT because the data points are now weighted by the inverse of their errors. After the solution for the principal component has been established, the secondary orbit can be established simply by solving for $k_{2}$. The orbital solution for the binary is listed in Table 5 and plotted in Fig. 6.

Inspection of the resulting fits shows that a solution with $v_{\text {orb }}=2.0754 \mathrm{day}^{-1}$ gives substantially the best result. Errors are estimated from a range of solutions based on an uncertainty in the orbital frequency $\left(\sim \pm 0.01 \mathrm{day}^{-1}\right)$. Thus these results are intended as an early indication of the dimensions of the binary PG 1544+488 rather than as a definitive solution.

\section{Discussion}

Since their identification in the PG survey, the puzzling nature of the rare helium-rich subdwarf $\mathrm{B}$, or sdOD, stars has been neglected, but not forgotten. Two groups have recently published independent analyses of the spectrum of PG $1544+488$, the star identified as the prototype by Heber et al. (1988), one based on medium-resolution blue-optical spectrum (Paper I) and supported by the IUE/UV flux distribution (Paper II), and the other based on the high-resolution far-ultraviolet spectrum (Lanz et al. 2004). Both studies obtained a radically different measurement of surface gravity $(\log g)$. The optical spectrum indicates a $\log g$ of 5.1 dex in cgs units, while the farultraviolet spectrum indicates $\log g=6.0$. Lanz et al. (2004) also found a very high rotational velocity $\left(v \sin i=100 \mathrm{~km} \mathrm{~s}^{-1}\right)$ and suggest that the discrepancy in $\log g$ is due to the use of different carbon abundances in the model atmospheres. This suggestion has not yet been tested because the discovery that PG $1544+488$ is a spectroscopic binary completely changes the basis for argument.
Table 5. Approximate orbital solution for PG 1544+488.

\begin{tabular}{rcl}
\hline \hline$P$ & $0.48 \pm 0.01$ & day \\
$\gamma$ & $-23 \pm 4$ & $\mathrm{~km} \mathrm{~s}^{-1}$ \\
$k_{1}$ & $57 \pm 4$ & $\mathrm{~km} \mathrm{~s}^{-1}$ \\
$k_{2}$ & $97 \pm 10$ & $\mathrm{~km} \mathrm{~s}^{-1}$ \\
\hline$q$ & $1.7 \pm 0.2$ & \\
$a \sin i$ & $1.47 \pm 0.13$ & $R_{\odot}$ \\
$M_{1} \sin ^{3} i$ & $0.114 \pm 0.021$ & $M_{\odot}$ \\
$M_{2} \sin ^{3} i$ & $0.067 \pm 0.011$ & $M_{\odot}$ \\
\hline
\end{tabular}

The WHT spectra reveal more than just radial velocities for two stellar components. They also show that the surfaces of both components are helium-rich; there is no significant line at $\mathrm{H} \alpha$ or $\mathrm{H} \beta$, while both components are apparent in HeI. The HeI profiles at $6678 \AA$ are not symmetric, the components have different line strengths and hence different luminosities. The line doubling is prominent at $6678 \AA$, measurable at $4921 \AA$, increasingly weaker by $4471 \AA$ and not seen in $4026 \AA$. This implies that the contribution of the weaker component to the total flux decreases towards shorter wavelength as a consequence of a difference in effective temperature. Thus, in the farultraviolet, the observed spectrum is dominated by the brighter and hotter component.

There are several consequences. First, existing analyses have been based on observations integrated over long intervals. The apparently large rotational velocity reported is a direct consequence of orbital velocity smearing. This smearing can be removed and the analyses should be repeated. Second, it is no surprise that analyses based on far-ultraviolet and optical spectra alone yield different results, since the observed line profiles are contaminated by different relative contributions from the secondary star. Analyses must take the binary nature of the system into account. Third, the interpretation of the evolutionary status of PG 1544+488, which requires it to be a single star, must be wrong. The measurements reported here demonstrate that PG $1544+488$ is almost certainly a product of close binary evolution.

However, there is already much that may be deduced about the nature of PG 1544+488. Unless the orbital inclination is extraordinarily high, both components must be of low mass, say $0.1-0.5 M_{\odot}$. Both are early-type stars with surfaces dominated by helium. Both stars must have evolved off the main-sequence and developed helium cores and there must have been at least one phase of mass transfer when the system was probably a common envelope binary. It seems that the entire hydrogenrich envelopes from both stars were removed at this stage, most probably by the ejection of the common envelope. This process would have been closely associated with the contraction of the orbit to its current value of just a few solar radii. The primary is also carbon-rich (Lanz et al. 2004), implying that it has undergone some helium-processing before this last mass transfer phase was completed. It also suggests that it must be above the 
minimum mass for helium ignition, placing a minimum value on the allowable orbital inclination.

These deductions give some clues to the possible evolution of PG $1544+488$. The dimensions of the observed system are typical of the dimensions of more conventional shortperiod sdB close binaries with WD companions (Maxted et al. 2001), although the mass function of the secondary may be on the low side. Han et al. (2002) argue that these systems are formed as the result of a common-envelope evolution. When the observed sdB star or, in the present case, the more massive helium star became a giant following core hydrogen exhaustion, its radius expanded until Roche Lobe overflow commenced. Providing the mass ratio $\left(M_{2} / M_{1}\right)$ is less than some critical value ( $q_{\text {crit }} \sim 1$; cf. Han et al. 2002), mass transfer is dynamically unstable and a common envelope will form. The secondary will then spiral in, transferring orbital energy to the envelope and causing it to be ejected. The quantity of hydrogen-rich material left on the surface of the $\mathrm{sdB}$ star is very small, and one can envisage that, in some circumstances, all of the hydrogen may be removed. It may even show some contamination from carbon produced in the core helium flash. The question then arises concerning the nature of the secondary. Since it is also observed to be helium-rich it must be hot and compact - a low-mass main-sequence solution can be ruled out. It must therefore be either a helium white dwarf or a lowmass helium main-sequence object. It is likely that it would have originally been the more massive star and that it would have lost its hydrogen envelope during a previous phase of Roche-lobe overflow. While this description is more conjecture than conclusion, it demonstrates that PG 1544+488 represents a very important test for models of close-binary evolution involving a common-envelope; further observations and more detailed analyses will be needed to refine these tests.

Meanwhile, it is interesting to compare PG $1544+488$ with other evolved stars. The mass of the secondary is likely to be less than $0.3 M_{\odot}$. There are several known binary systems in which at least one component is a highly-evolved subluminous star, with a mass of $\sim 0.3 M_{\odot}$; e.g. HD185510 (Jeffery \& Simon 1997), HD128220 (Howarth \& Heber 1990), HD188112 (Heber et al. 2003). In these cases, the secondary is believed to be a helium-core white dwarf progenitor. A similar situation probably holds for the secondary of PG $1544+488$.

Recently, Lisker et al. (2004) have discovered a very similar system HE 0301-3039 from the VLT/UVES SPY survey (Napiwotski et al. 2001). In this case, the line profile splits into more symmetric components and are clearly resolved at $3865 \AA$ as well as $5048 \AA$, suggesting the components to be of more similar temperature and luminosity than in the case of PG 1544+488. It will be interesting to compare these two systems in detail as more observations are obtained.

Whilst it is clear that PG $1544+488$ is a binary, the question of whether other He-sdB stars are also binaries is not. As discussed in Paper II, the group is quite heterogeneous, including relatively hydrogen-rich stars such as JL 87 (EC 21435-7634) and carbon-poor extremely helium-rich stars such as LB 1766. Whether any or all of these are binaries will have to be determined from a more extensive and systematic radial velocity survey.

\section{Conclusions}

We have measured radial velocities for the prototype heliumrich subdwarf B star - PG 1544+488 - using spectra obtained with the INT, WHT and FUSE. The high-resolution WHT spectra show line-splitting characteristic of a spectroscopic binary, with HeI lines in both components and hydrogen lines in neither. Further observations will be necessary to accurately determine the orbital period, but it is likely to be $0.48 \mathrm{~d}$. The low mass functions indicate that PG 1544+488 comprises two lowmass helium-rich subluminous stars separated by a few solar radii. Consequently it would appear to be a highly evolved system which has passed through at least one common-envelope phase. The entire hydrogen envelope has been lost from both stars. We conjecture that this occurred through common envelope ejection shortly after helium ignition in what is now seen as the more massive component. The less massive component is either a helium white dwarf or a very low mass helium mainsequence star.

Acknowledgements. This research is supported by a grant to the Armagh Observatory from the Northern Ireland Department of Culture, Arts and Leisure (DCAL) and by PPARC through the award of telescope time and travel grants. The authors wish to thank the referee for a helpful and timely report. They also acknowledge the data analysis facilities provided by the Starlink Project which is run by CCLRC on behalf of PPARC. This research has made use of NASA's ADS. This publication makes use of data products from the MAST archives.

\section{References}

Ahmad, A., \& Jeffery, C. S. 2003, A\&A, 402, 335

Ahmad, A., \& Jeffery, C. S. 2004, A\&A, 413, 323

Brown, T. M., Sweigart, A. V., Lanz, T., Landsman, W. B., \& Hubeny, I. 2001, ApJ, 562, 368

Dudley, R. E., \& Jeffery, C. S. 1990, MNRAS, 247, 400

Green, R. F., Schmidt, M., \& Liebert, J. 1986, ApJS, 61, 304

Han, Z., Podsiadlowski, Ph., Maxted, P. F. L., Marsh, T. R., \& Ivanova, N. 2002, MNRAS, 336, 449

Heber, U., Dreizler, S., de Boer, K. S., Moehler, S., \& Richtler, T. 1988, AGAb, 1, 16

Heber, U., Edelmann, H., Lisker, T., \& Napiwotzki, R. 2003, A\&A, 411, L477

Högbom, J. A. 1974, A\&AS, 15, 417

Howarth, I. D., \& Heber, U. 1990, PASP, 102, 912

Howarth, I. D., Murray, J., Mills, D., \& Berry, D. S. 1998, Starlink User Note, 50

Iben, I. J., \& Tutukov, A. V. 1986, ApJ, 311, 753

Jeffery, C. S., \& Simon, T. 1997, MNRAS, 286, 487

Lanz, T., Brown, T. M., Sweigart, A. V., Hubeny, I., \& Landsman, W. B. 2004, ApJ, 602, 342

Lisker, T., Heber, U., Napiwotzki, R., et al. 2004, Ap\&SS, in Extreme Horizontal Branch Stars and Related Objects, ed. P. F. L. Maxted, in press

Maxted, P. F. L., Heber, U., Marsh, T. R., \& North, R. C. 2001, MNRAS, 326, 1391

Moehler, S., Heber, U., \& Durrell, P. R. 1997, A\&A, 317, L83

Moehler, S., Sweigart, A. V., Landsman, W. B., \& Dreizler, S. 2002, A\&A, 395, 37

Napiwotzki, R., Christlieb, N., Drechsel, H., et al. 2001, Astron. Nachr., 322, 411

Saio, H., \& Jeffery, C. S. 2000, MNRAS, 313, 671 